\title{
The role of pelvic radiotherapy in stage IV rectal cancer-still working in the dark!
}

\author{
Rob Glynne-Jones \\ Radiotherapy Department, Mount Vernon Centre for Cancer Treatment, Mount Vernon Hospital, Northwood, UK \\ Correspondence to: Rob Glynne-Jones, MD, FRCR. Mount Vernon Centre for Cancer Treatment, Rickmansworth Road, Northwood, UK. \\ Email: rob.glynnejones@nhs.net. \\ Comment on: Agas RAF, Co LBA, Jacinto JCKM, et al. Neoadjuvant Radiotherapy Versus No Radiotherapy for Stage IV Rectal Cancer: a Systematic \\ Review and Meta-analysis. J Gastrointest Cancer 2018;49:389-401.
}

Received: 01 October 2018; Accepted: 05 December 2018; Published: 14 December 2018.

doi: $10.21037 /$ tro.2018.12.03

View this article at: http://dx.doi.org/10.21037/tro.2018.12.03

Approximately $20 \%$ of patients with colorectal cancer (CRC) are found to have metastatic colorectal cancer (mCRC) at diagnosis, but only $20 \%$ of these are usually considered to be potentially resectable (de Haas 2011) (1). Early stage disease in rectal cancer confers a relatively favourable prognosis, but stage IV or metastatic disease is associated with a five-year survival of only $10-15 \%$, despite major advances in multimodality treatment. Individual outcomes are influenced by the site and number of metastases and the specific metastatic sites involved. Patients with rectal primaries are also more likely to present with synchronous lung metastases than patients with colon cancer (Robinson 2018) (2), which then can lead to a different natural history with bone and brain metastases.

The optimal strategy for patients with rectal cancer presenting with synchronous metastases remains highly controversial because the survival is poor and several modalities of treatment are available (surgery/radiotherapy/ chemotherapy) all of which can be potentially combined. Traditionally, resection of the primary tumour was performed first in order to palliate local symptoms and prevent future complications such as obstruction and haemorrhage. Yet the ease and rapidity of recovery after laparoscopic stoma formation, calls initial radical resection into question. Debate also continues to simmer as to the role of pelvic radiotherapy (RT) in this setting; what type of RT (short course or chemoradiation (CRT), when to give it, and who ought to have it (Pfeiffer 2018) (3). In fournal of Gastrointestinal Cancer a recently published meta-analysis (Agas 2018) (4) shines some light on these questions and highlights the ongoing challenges.

This meta-analysis highlights the limited evidence in that there were only 8 studies considered eligible. The Dutch TME trial is the only randomised phase III trial in this setting, but had just 95 patients with stage IV disease. In addition, there were 5 retrospective cohorts (only 420 patients) and 2 population-based studies from the Surveillance, Epidemiology, and End Results (SEER) database and the Swedish Rectal Cancer Registry (SRCR). These 2 latter registries provided the majority of patients $5,612 / 6,170(90 \%)$ in the meta-analysis (Hosseinali Khani 2012, Wu 2017) $(5,6)$.

The Pooled analysis of the 8 studies showed significantly better local recurrence-free survival (LRFS) after RT versus no RT with a risk ratio (RR) of 1.15 (95\% CI, 1.01-1.31, $\mathrm{P}=0.03)$. Interestingly the results show metastatic disease 'per se' confers an increased risk of local recurrence in multivariate analysis (as it does in breast cancer), suggesting a hazard ratio of 16.2 (95\% CI, 5.4-48.6, $\mathrm{P}<0.001)$ and $20.26(95 \% \mathrm{CI}, 7.43-55.28, \mathrm{P}<0.001)$ at 2 - and 5 -year follow-up, respectively.

Traditional concepts regarding the risk of local recurrence in locally advanced rectal cancer (LARC) have evolved differently in different countries to the point where some use short course preoperative radiotherapy (SCPRT) using $5 \times 5$ Gy, and others CRT and with diverse selection criteria. Historically, because of poor surgical techniques using blunt dissection, a high loco-regional recurrence rate up to $30 \%$ in some studies was reported after radical surgery alone, which would be considered 
unacceptable today. The resultant symptoms including intractable pelvic pain, which, once entrenched, were extremely difficult to palliate, influenced a generation to employ pelvic RT in the preoperative setting for virtually all patients with rectal cancer-irrespective of stage (Kapiteijn 2001) (7). Since publication of the German $\mathrm{CAO} / \mathrm{ARO} / \mathrm{AIO}$ trial preoperative CRT has become the standard of care for LARC.

However, acknowledgement of the long-term adverse consequences of radiation and improved imaging in terms of high-resolution magnetic resonance imaging (MRI) and computerized tomography (CT) and positron emission tomography combined with CT (PET/CT) have allowed a more individualized management rather than 'a one size fits all' approach. Pelvic MRI of the pelvis, provides an accurate imaging of primary tumour its proximity to the mesorectal fascia and hence the risk of failing to achieve an R0 resection will detect metastatic disease. PET/CT may also be helpful because $10-20 \%$ of more advanced cases with extramural vascular invasion have the initial clinical stage refined by metastatic disease. Patients with clinical stage III rectal cancer are frequently advised to receive preoperative RT based on randomised trial data. Yet, many questions whether patients with potentially curable metastatic rectal cancer may also benefit from RT/CRT to the same extent.

What this study does not clarify is whether CRT or SCPRT is more effective or whether both schedules are equivalent. It would be interesting in future to have an individual participant data (IPD) meta-analysis examining if there is any difference between SCPRT using $5 \times 5$ Gy and CRT.

In addition, there is no guidance on the optimal sequencing of chemotherapy/radiotherapy and surgery. The Dutch TME study randomised between $5 \times 5$ Gy short course preoperative radiotherapy and immediate surgery versus surgery alone. Patient and treatment characteristics were well balanced between the two intervention groups, but there were only 95 patients with stage IV disease. Analysis showed a marked difference in 2- and 5-year local recurrence $10.1 \%$ versus $23.8 \%$, and $15.9 \%$ versus $26.9 \%$, for RT versus no RT respectively, but this difference was not statistically significant (Kapiteijn 2001, Peters 2007) $(7,8)$. The relatively few included patients with stage IV ( $\mathrm{n}=95)$ may have influenced the chance of showing a statistically significant difference in standard oncological outcomes such as LR, DFS and OS between the two groups.

One unexpected finding from the meta-analysis is that the pooled analysis data suggest that there may be a benefit in 5-year OS with RT in patients with stage IV disease. The usual caveats apply regarding interpretation of retrospective studies and population-based data only, since patients with more limited oligo-metastatic disease and better performance status may be more likely to be considered appropriate for RT. This suggestion that OS is improved also goes against all recent data in both SCPRT and CRT studies in the aftermath of the Swedish Rectal Cancer Trial (SRCT 1997) (9), where improvements in survival have not been reported. Randomised trials show improvements in local control at 5 years even as much as $15 \%$ after CRT have no significant impact on OS (Braendengen 2018) (10). Improved survival is unlikely to reflect a lower local recurrence, rather the fact that the majority of patients die from distant metastases. However, if the findings are real, other explanations may be required.

The primary rectal tumour can be symptomatic or asymptomatic, and the rationale for delivering RT has been to avoid obstruction, bleeding and the requirement for a palliative colostomy. If the liver and primary tumour can both be resected radiotherapy or chemoradiation has usually been given to reduce the subsequent risk of local recurrence. Proponents of short-course preoperative radiotherapy (SCPRT) using $5 \times 5$ Gy as a component of multimodality treatment argue that SCPRT can be followed by systemic doses of chemotherapy within 10 days of completion of SCPRT. The advantage of this strategy includes ensuring prompt delivery and maintaining the dose intensity of systemic chemotherapy. Some of the best reported results using this strategy for synchronous potentially resectable metastases and rectal cancer have recently been updated (Van Dijk 2013) (11). This trial used $5 \times 5$ Gy followed by capecitabine. Oxaliplatin and bevacizumab. The 2-year recurrence rate was $64 \%(95 \%$ CI, $49.8-84.5 \%)$. At 8.1 years $16 / 50$ patients $(32.0 \%)$ were still alive and $14(28 \%)$ were disease-free. Of 36 patients who received radical treatment, only $2 / 36(5.6 \%)$ had a local recurrence (Van Dijk 2013) (11). The original shortterm efficacy was influential in the design of the Rectal Cancer and Preoperative Induction Therapy Followed by Dedicated Operation (RAPIDO) trial (Nilsson 2013) (12), which assigns patients with locally advanced rectal cancer to SCPRT followed by six cycles of capecitabine and intravenous oxaliplatin, and then TME versus preoperative long-course CRT followed by TME.

There is evidence that besides damaging and killing cancer cells, radiation may destroy the adjacent tumour- 
protective stromal microenvironment, with particular responses reflecting changes in local immune cells (Demaria 2016) (13). Fractionated radiation appears to be more effective in the clinical setting than single doses in triggering anticancer immune responses (Formenti 2017) (14). So $5 \times 5$ Gy may be an effective method or producing neoantigens-particularly when followed immediately by oxaliplatin based systemic chemotherapy. The Polish 2 trial (Bujko 2016) (15) showed a survival advantage of $5 \times 5$ Gy followed by FOLFOX compared to CRT.

Administration of systemic therapy is usually delayed if the primary tumour is initially resected, and occasionally surgical complications including pelvic sepsis can produce such extensive delay that chemotherapy is never given. It is possible that radiotherapy offers a sense of security and allows full delivery of chemotherapy at an early point in time and reduces the risk of local problems from the tumour itself such as obstruction which require emergency surgery and inhibit further subsequent chemotherapy because of a drop in PS. In the Swedish registry data, survival for patients with stage IV rectal cancer increased over time despite doubling the proportion of patients, who did not undergo any surgical treatment.

However, the value of RT treatment in this setting remains controversial and evidence to guide decisions regarding the optimal approach in this population remains sparse. There are no specific trials randomising CRT and chemotherapy against chemotherapy alone for these patients. In practice, CRT is often recommended by the multidisciplinary team (MDT) in patients where the metastases are considered resectable or potentially convertible, and often omitted where the metastases are assessed as never likely resectable so as to avoid unnecessary toxicity from CRT. MDTs are also keen to deliver CRT for younger fitter patients with low T4 lesions, and oligometastases because of concerns to maintain optimal local control (Lin 2012) (16).

There is also an innate bias in retrospective studies such that RT is more likely to be administered for patients with predominantly local symptoms and upfront chemotherapy for patients when systemic symptoms from distant metastases are uppermost.

ESMO rectal cancer guidelines are not explicit and allow for both CRT and SCPRT in curative approaches, and SCPRT alone in the palliative setting (Glynne-Jones 2017) (17). The ESMO guidelines for rectal cancer refer to ESMO consensus guidelines for the management of patients with metastatic colorectal cancer (Van Cutsem 2016) (18), which in turn advise that if the primary and metastases are easily resectable, that perioperative FOLFOX should be administered before and after liver resection (Van Cutsem 2016) (18). In selected cases, the primary may be resected concurrently. Recommendations are to administer this chemotherapy for a total of at least 6 months, but do not discuss the role of either CRT or SCPRT. According to the National Comprehensive Cancer Network (NCCN) guidelines (3. 2018) (NCCN guidelines) (19), there are 4 different recommendations for 4 different scenarios. The guidelines are clearly defined and quite prescriptive. They consider lung and liver metastases separately, depending on whether they are resectable or not and recommend upfront systemic chemotherapy followed by chemoradiotherapy (CRT), but $5 \times 5$ Gy is preferred if the radial margin is predicted clear on MRI (Recommendation 8). For unresectable metastases a similar algorithm recommends $5 \times 5$ Gy in preference (Recommendation 9).

It is surprising how little evidence is available apart from registry /population data which inevitably gives one no insight into why some patients with stage IV rectal cancer receive radiotherapy and others do not. Current clinical practice is largely based on evidence from non-randomised, retrospective, population-based, but not even propensitymatched studies. (The Swedish study analysed by different age groups and different time periods (1995-1998, 1999-2002, 2003-2006) to determine changes in treatment strategies) (Hosseinali Khani 2012) (5). Hence the final period describes practice at least 12 years ago, when pre-treatment staging was much less effective and pelvic MRI was rarely used. Postoperative mortality rates were much higher than today. Options for chemotherapy and biological agents were not so well understood. Interestingly, in the final period of this analysis only $52 \%$ of patients that had a bowel resection and $25 \%$ of patients who did not undergo surgery received chemotherapy and we have no data on the intensity of this chemotherapy. The SEER data is more up to date and identified patients 2004-2014.

In our current era with the widespread integration of the MDT, more individualised and safer surgery for both the primary and metastatic disease is available. More appropriate and more selective use of more intensive chemotherapy in a larger proportion of this population may also be able to improve outcomes.

Previous trials examining the role of surgery to the primary or metastases in stage IV colorectal cancer (as opposed to specifically rectal cancer) have singularly failed to recruit because clinicians appear to lack equipoise either 
as regards the primary management (NCT01086618) or metastatic disease (NCT01056809) (NCT01106261). I suspect that equipoise regarding the utility of pelvic radiotherapy, would also be often lacking in the setting of rectal cancer with synchronous metastases.

Nevertheless, prospective and randomised studies in this population are still required-and in many ways are even more crucial-to establish the best therapeutic sequences, to demonstrate the optimal RT dose and fractionation ( $5 \times 5$ Gy or long course chemoradiation), and to provide some data in terms of quality of life (QoL). The design and conduct of these trials are bound to be challenging.

\section{Acknowledgments}

Funding: None.

\section{Footnote}

Provenance and Peer Review: This article was commissioned and reviewed by the Section Editor Hsin Hua Nien (Department of Radiation Oncology, Cathay General Hospital, Taipei, Taiwan).

Conflicts of Interest: Rob Glynne-Jones has received honoraria for lectures and advisory boards and has been supported in attending international meetings in the last 5 years by Merck, Pfizer, Sanofi-Aventis and Roche. He has also in the past received unrestricted grants for research from Merck-Serono, Sanofi-Aventis and Roche. He is principal investigator of a randomised phase II neoadjuvant chemotherapy study in the UK called 'BACCHUS'.

Etbical Statement: The author is accountable for all aspects of the work in ensuring that questions related to the accuracy or integrity of any part of the work are appropriately investigated and resolved.

Open Access Statement: This is an Open Access article distributed in accordance with the Creative Commons Attribution-NonCommercial-NoDerivs 4.0 International License (CC BY-NC-ND 4.0), which permits the noncommercial replication and distribution of the article with the strict proviso that no changes or edits are made and the original work is properly cited (including links to both the formal publication through the relevant DOI and the license). See: https://creativecommons.org/licenses/by-nc$\mathrm{nd} / 4.0 /$.

\section{References}

1. de Haas RJ, Wicherts DA, Andreani P, et al. Impact of Expanding Criteria for Resectability of Colorectal Metastases on Short- and Long-Term Outcomes After Hepatic Resection. Ann Surg 2011;253:1069-79.

2. Robinson JR, Newcomb PA, Hardikar S, et al. Stage IV colorectal cancer primary site and patterns of distant metastasis. Cancer Epidemiol 2017;48:92-5.

3. Pfeiffer P, Gruenberger T, Glynne-Jones R. Synchronous liver metastases in patients with rectal cancer: can we establish which treatment first? Ther Adv Med Oncol 2018;10:1758835918787993.

4. Agas RAF, Co LBA, Jacinto JCKM, et al., Neoadjuvant Radiotherapy Versus No Radiotherapy for Stage IV Rectal Cancer: a Systematic Review and Meta-analysis. J Gastrointest Cancer 2018;49:389-401.

5. Hosseinali Khani M, Pahlman L, Smedh K. Treatment strategies for patients with stage IV rectal cancer: a report from the Swedish Rectal Cancer Registry. Eur J Cancer 2012;48:1616-23.

6. Wu Y, Liu H, Du XL, et al. Impact of neoadjuvant and adjuvant radiotherapy on disease-specific survival in patients with stages II-IV rectal cancer. Oncotarget 2017;8:106913-25.

7. Kapiteijn E, Marijnen CA, Nagtegaal ID, et al. Preoperative radiotherapy combined with total mesorectal excision for resectable rectal cancer. $\mathrm{N}$ Engl $\mathrm{J}$ Med 2001;345:638-46.

8. Peeters KC, Marijnen CA, Nagtegaal ID, et al. The TME trial after a median follow-up of 6 years: increased local control but no survival benefit in irradiated patients with resectable rectal carcinoma. Ann Surg 2007;246:693-701.

9. Swedish Rectal Cancer Trial, Cedermark B, Dahlberg M, et al. Improved survival with preoperative radiotherapy in resectable rectal cancer Swedish Rectal Cancer Trial. N Engl J Med 1997;336:980-7.

10. Brændengen M, Glimelius B. Preoperative radiotherapy or chemoradiotherapy in rectal cancer - Is survival improved? An update of the "Nordic" LARC study in non-resectable cancers. Radiother Oncol 2018;127:392-5.

11. van Dijk TH, Tamas K, Beukema JC, et al., Evaluation of short-course radiotherapy followed by neoadjuvant bevacizumab, capecitabine, and oxaliplatin and subsequent radical surgical treatment in primary stage IV rectal cancer. Ann Oncol 2013;24:1762-9.

12. Nilsson PJ, van Etten B, Hospers GA, et al. Short-course radiotherapy followed by neo-adjuvant chemotherapy in 
locally advanced rectal cancer -- the RAPIDO trial. BMC Cancer 2013;13:279.

13. Demaria S, Coleman CN, Formenti SC. Radiotherapy: Changing the Game in Immunotherapy. Trends Cancer 2016;2:286-94.

14. Formenti SC. Optimizing Dose Per Fraction: A New Chapter in the Story of the Abscopal Effect? Int J Radiation Oncol Biol Phys 2017;99:677-9.

15. Bujko K, Wyrwicz L, Rutkowski A, et al. Long-course oxaliplatin-based preoperative chemoradiation versus $5 \times 5$ Gy and consolidation chemotherapy for cT4 or fixed cT3 rectal cancer: results of a randomized phase III study. Ann Oncol 2016;27:834-42.

16. Lin JK, Lee LK, Chen WS, et al. Concurrent

doi: $10.21037 /$ tro.2018.12.03

Cite this article as: Glynne-Jones R. The role of pelvic radiotherapy in stage IV rectal cancer-still working in the dark! Ther Radiol Oncol 2018;2:62. chemoradiotherapy followed by metastasectomy converts to survival benefit in stage IV rectum cancer. J Gastrointest Surg 2012;16:1888-96.

17. Glynne-Jones R, Wyrwicz L, Tiret E, et al. Rectal cancer: ESMO Clinical Practice Guidelines for diagnosis, treatment and follow-up. Ann Oncol 2017;28:iv22-40.

18. Van Cutsem E, Cervantes A, Adam R et al. ESMO consensus guidelines for the management of patients with metastatic colorectal cancer. Ann Oncol 2016;27:1386-422.

19. NCCN Clinical Practice Guidelines in Oncology (NCCN Guidelines $\left.{ }^{\circledR}\right)$ Rectal Cancer Version 3.2018 — August 7, 2018 NCCN.org last accessed 28 September 2018. Available online: https://www.nccn.org 